\title{
Trichophoromyia auraensis is a putative vector
}

\section{Carolina Bioni Garcia Teles ${ }^{1,2,5,6 /+}$, Felipe Arley Costa Pessoa ${ }^{3}$, Jansen Fernandes Medeiros ${ }^{1,2,6}$, Luís Marcelo Aranha Camargo ${ }^{4,5,6}$}

\author{
${ }^{1}$ Fundação Oswaldo Cruz-Fiocruz, Porto Velho, RO, Brasil \\ ${ }^{2}$ Universidade Federal de Rondônia, Porto Velho, RO, Brasil \\ ${ }^{3}$ Fundação Oswaldo Cruz-Fiocruz, Instituto Leônidas e Maria Deane, Manaus, AM, Brasil \\ ${ }^{4}$ Universidade de São Paulo, Instituto de Ciências Biomédicas, Monte Negro, RO, Brasil \\ ${ }^{5}$ Faculdade São Lucas, Porto Velho, RO, Brasil \\ ${ }^{6}$ Instituto Nacional de Epidemiologia na Amazônia Ocidental, Porto Velho, RO, Brasil
}

The sandfly Trichophoromyia auraensis has recently evolved as a proven vector of Leishmania (Viannia) endemic to state of Acre in the north of Brazil. This note is intended to propose a correction in the report of the first occurrence of natural infection of Leishmania (Viannia) in this species. We and the other scientific groups reinforced that Tr. auraensis is a possible vector involved in the transmission of American cutaneous leishmaniasis in Acre, Brazil.

Key words: Leishmania - disease vectors - Acre - Brazil - Trichophoromyia auraensis

About the article: First description of Leishmania (Viannia) infection in Trichophoromyia auraensis (Psychodidae: Phlebotominae) in the transmission area of American cutaneous leishmaniasis in Acre state, Amazon Basin, Brazil.

American cutaneous leishmaniasis (ACL) is a disease highly endemic to the state of Acre, Brazil. This state is located on the Brazilian border and is surrounded by Peru and Bolivia, both of which have a high number of ACL cases reported. The state of Acre (Brazil) was the unit of the federation with the highest incidence of ACL in 2010, reaching 141.64 cases per 100,000 inhabitants. However, very few studies have been conducted on the development of sandfly ecology in this state. A quick search on Scopus demonstrated that only seven studies researching on this topic in the state of Acre have been published thus far. Valdivia et al. (2012), who found Trichophoromyia auraensis (Mangabeira) infected with Leishmania (Viannia) in Madre Dios, the Peruvian state that borders Acre, indicated that it is the main vector of Leishmania (Viannia) in the Southwest Amazon Basin. In Acre, both the microregion of Rio Branco and a municipality (Assis Brasil) located in the microregion of Brasileia, are considered endemic areas of epidemiological relevance for ACL. According to the Notifiable Diseases Information System (SINAN 2014), 387 cases were reported in the municipality between 2007 and 2013 , corresponding to an annual mean detection coefficient of $93 \mathrm{ACL}$ cases per 10,000 inhabitants.

doi: 10.1590/0074-02760170024

+ Corresponding author: carbioni2004@gmail.com

Received 20 January 2017

Accepted 10 February 2017
Owing to its epidemiological importance, our research groups chose this area for conducting some epidemiological studies focusing on the ecological aspects of sandflies and Leishmania. In this region, 13 new occurrences were registered and two new species of sandflies were described (Teles et al. 2013, Oliveira et al. 2015). Moreover, the species of Leishmania in human cases were characterised by polymerase chain reaction (PCR) (Teles et al. 2015).

In July 2016, our team published an epidemiological study conducted on almost seven thousand collected sandflies in Memórias do Instituto Oswaldo Cruz (MIOC) (Teles et al. 2016). The females were grouped by pools and examined for the DNA tracks of Leishmania. We found that specimens of Lutzomyia davisi and $L u$. auraensis/Lu. ruifreitasi contained the DNA tracks of Leishmania from the L. braziliensis complex at high concentrations. We registered this as the first report of the infection of $L u$. auraensis/Lu. ruifreitasi by $L$. (V.) guyanensis and L. (V.) braziliensis in Brazil.

This note is to make a scientific correction; recently, in MIOC, January 2017, de Araujo-Pereira et al. (2017) assigned the first description of Leishmania $(V$.$) infection$ in Tr. auraensis in the Rio Branco municipality of Acre state. The note of de Araujo-Pereira et al. (2017), despite not inedited to the occurrence, is important because it reinforces that it is possible to find the infected populations of Tr. auraensis in some hundreds of kilometres of the endemic regions considered as ACL hot spots such as Assis Brasil, in Acre. This species also occurs in other Brazilian states such as Amazonas, Pará, Rondônia and Mato Grosso, which are characterised by high population, highways, intense deforestation, and intense migration caused by economical attractiveness. Therefore, these states should be studied more deeply to understand the role of this sandfly in the transmission of ACL. 


\section{REFERENCES}

de Araujo-Pereira T, de Pita-Pereira D, Boité MC, Melo M, da CostaRego TA, Fuzari AA, et al. First description of Leishmania (Viannia) infection in Evandromyia saulensis, Pressatia sp. and Trichophoromyia auraensis (Psychodidae: Phlebotominae) in a transmission area of cutaneous leishmaniasis in Acre state, Amazon Basin, Brazil. Mem Inst Oswaldo Cruz. 2017; 112(1): 75-8.

Oliveira AFJ, Teles CBG, Medeiros FM, Camargo LMA, Pessoa FAC. Description of Trichophoromyia ruifreitasi, a new phlebotomine species (Diptera, Psychodidae) from Acre state, Brazilian Amazon. Zookeys. 2015; 526: 65-73.

SINAN - Sistema de Informações de Agravos de Notificação[Internet]. [cited 2014 Set 28]. 2014. Available from: http://dtr2004.saude.gov.

Teles CBG, dos Santos APA, Freitas RA, de Oliveira AFJ, Ogawa GM, Rodrigues MS, et al. Phlebotomine sandfly (Diptera: Psychodidae) diversity and their Leishmania DNA in a hot spot of American Cutaneous Leishmaniasis human cases along the Brazilian border with Peru and Bolivia. Mem Inst Oswaldo Cruz. 2016; 111(7): 423-32.

Teles CBG, Freitas RA, Oliveira AFJ, Ogawa GM, Cavalcante EA, Medeiros JF, et al. Description of a new phlebotomine species (Diptera: Psychodidae, Phlebotominae) and new records of sand flies from the state of Acre, Northern Brazil. Zootaxa. 2013; 3609: 85-90.

Teles CBG, Medeiros JF, Santos APA, de Freitas LAR, Katsuragawa TH, Cantanhêde LM, et al. Molecular characterization of American cutaneous leishmaniasis in the tri-border area of Assis Brasil, Acre state, Brazil. Rev Inst Med Trop São Paulo. 2015; 57(4): 343-7.

Valdivia HO, de los Santos MB, Fernandez R, Baldeviano GC, Zorri1la VO, Vera H, et al. Natural Leishmania infection of Lutzomyia (Trichophoromyia) auraensis in Madre de Dios, Peru, detected by a fluorescence resonance energy transfer-based real-time polymerase chain reaction. Am J Trop Med Hyg. 2012: 87(3): 511-7.

\section{REPLY}

Comments about the finding of Trichophoromyia auraensis (Mangabeira 1942) sandfly specimens infected by Leishmania (Viannia) parasites in Acre state

Since 2011, our group has been conducting field studies in the state of Acre, trying to contribute with new findings about phlebotomine sandflies fauna and potential leishmaniasis vectors in the state. In an epidemiological context, Amazon is undergoing rapid changes in environmental conditions that directly affect the population of sandfly vectors and therefore disease transmission. Although considered a region with an increasing incidence of cutaneous leishmaniasis, knowledge of the geographical distribution of phlebotomine sandflies associated with disease transmission is still scarce for some regions, such as Acre state, where the cutaneous manifestation of the disease reached high prevalence in the last years (Martins \& Silva 1964, Arias \& Freitas 1982, Arias et al. 1984, Silva-Nunes et al. 2008, Azevedo et al. 2008, Teles et al. 2013, de Araujo-Pereira et al. 2014, Oliveira et al. 2015).

From 2011 to 2012, we centralised the sandfly captures in residential areas within forested regions and recreational areas of Rio Branco municipality, the capital of Acre, and found a very diverse fauna in urban and peri urban areas of the city, as diverse as that encountered in areas of primary forest. A total of 16 Phlebotominae genera and 23 species was taxonomically identified, being Trichophoromyia auraensis and Nyssomyia whitmani the most abundant species in both forested and peridomiciliary environments. Taking into account the high prevalence of Th. auraensis in Rio Branco (more than $50 \%$ of the total captured insects), we reported in 2014, this species as a putative vector in the region (de Araujo-Pereira et al. 2014).

The manuscript by Oliveira et al. (2015) describes Trichophoromyia ruifreitasi, a new phlebotomine sandfly species from Acre, morphologically similar to $T h$. auraensis, that was identified between male specimens collected in the municipality of Assis Brasil (Oliveira et al. 2015, Teles et al. 2016). As mentioned by Oliveira et al. (2015), "This species is similar to Th. auraensis (Mangabeira), but the two species can be easily differentiated by the distribution of setae on their parameres, and by the presence of a dorsal lobe in the parameres of the new species". In this study, the authors detailed the morphological characteristics of male specimens of $T h$. ruifreitasi identified in the municipality of Assis Brasil (Oliveira et al. 2015). In contrast to the investigation by Teles et al. (2016) performed in the municipality of Assis Brasil, in the study areas of Rio Branco, we identified all male specimens of the Trichophoromyia genus as Th. auraensis (de Araujo-Pereira et al. 2014). The study by Oliveira et al. (2015) already mention our investigation about the sandfly fauna in Rio Branco, in which we report Th. auraensis as a putative vector in this Amazonian region (de Araujo-Pereira et al. 2014). Valdivia et al. (2012) were the first to identify Th. auraensis as a natural carrier of Leishmania (Viannia) lainsoni and Leishmania (Viannia) braziliensis in Madre de Dios, an Amazon region in Peru bordering the state of Acre. Similar to that observed in Rio Branco, in this region of Peru, Th. auraensis was also the most abundant sandfly species $(63 \%)$, and the minimum infection prevalence was estimated to be $0.6 \%$ by kDNA-polymerase chain reaction (kDNA-PCR) performed in insect pools. Using a FRET-based real time PCR, the authors identified four $T h$. auraensis pools positive for $L$. (V.) lainsoni and $L$. (V.) braziliensis (Valdivia et al. 2012).

Proceeding with our investigation in Rio Branco, we further analysed a sampling of non-blood-fed female sandflies for the presence of Leishmania DNA in each insect, individually. The choice for performing the molecular diagnosis separately, by individual specimen instead of pools of insects, was due to the complexity and diversity of the sandfly fauna in the Amazon Basin. Using a multiplex PCR directed simultaneously to Leishmania kDNA and to the IVS6 cacophony gene region in neotropical sandflies, coupled to hybridisation with a $L$. (Viannia) biotinylated probe (de Pita-Pereira et al. 2005), total positivity of $6.94 \%$ was found in the municipality of Rio Branco. The molecular characterisation of Leishmania spp. was performed by sequencing the $234 \mathrm{bp}$ fragment obtained by amplifying a region of the $h s p 70$ gene from each positive sample, previously assayed for the presence of Leishmania kDNA (da Graça et al. 2012). L. (V.) braziliensis DNA was identified in Th. auraensis, Evandromyia saulensis and Pressatia sp. individuals. For the other positive samples of Th. auraensis and $E v$. saulensis, the identification was restricted to the Viannia genus. These data were recently published in Memórias do Instituto Oswaldo Cruz (de Araujo-Pereira et al. 2017). The finding of L. (Viannia) DNA in two Ev. saulensis, with the confirmation of L. braziliensis in one specimen, corresponds to the first record of possible infection associated with this sandfly. Moreover, 
we registered in Rio Branco, Acre state, Th. auraensis and Pressatia sp. infected by L. (Viannia) parasites (in three out of nine positive Th. auraensis individuals and in the only positive Pressatia sp., the genetic material was confirmed as L. braziliensis). The role of these species as vectors of parasites responsible for New World tegumentary leishmaniasis remains to be established for better understanding the risk of the disease transmission in the Neotropics (de Araujo-Pereira et al. 2017).

In our opinion, both studies (Teles et al. 2016, de Araujo-Pereira et al. 2017) were published in very close periods, almost in parallel. Our manuscript was submitted in June 2016 and the one by the group of Teles was published in July 2016, during the peer-revision process of our manuscript.

Finally, we highlighted differences on some technical and critical aspects between the two studies. In Teles et al. (2016), the collected sandfly females from Assis Brasil, Acre state, were assembled in groups (220 specimens per group), and each pool was composed by the same species, date and place of capture. Because females of Th. auraensis and Th. ruifreitasi are probably indistinguishable, the authors named them $T h$. auraensis/Th. ruifreitasi [Lu. auraensis/Lu. ruifreitasi, according to Young and Duncan (1994)]. Teles et al. (2016) found a minimal infection prevalence of $2.05 \%$ in $\mathrm{Lu}$. auraensis/Lu. ruifreitasi (16 out of 58 pools) by parasites from the L. braziliensis complex. If one considers the limitation to morphologically differentiate between sandfly females of both species, our study in Rio Branco was advantageous, since there was no overlap among $T h$. auraensis and Th. ruifreitasi in the study areas. All male specimens of the Trichophoromyia genus were previously identified as Th. auraensis (de Araujo-Pereira et al. 2014), and were individually analysed for the presence of Leishmania DNA (de Araujo-Pereira et al. 2017).

In general, both studies (Teles et al. 2016, de AraujoPereira et al. 2017) are complementary, being performed in two municipalities of the state of Acre with high prevalence of cutaneous disease and thus bringing important contribution in demonstrating the diversity of sandfly species with potential involvement in the cutaneous leishmaniasis transmission cycle in the investigated areas.

\section{REFERENCES}

Arias JR, Freitas RA, Barrett TV. A new sandfly in the subgenus Nyssomyia (Diptera, Psychodidae) from the Amazon Basin of Brazil. Mem Inst Oswaldo Cruz. 1984; 79: 325-7.

Arias JR, Freitas RA. The known geographical distribution of sandflies in the state of Acre, Brazil (Diptera: Psychodidae). Acta Amaz. 1982; 12: 401-8.

Azevedo ACR, Costa SM, Pinto MCG, Souza JL, Cruz HC, Vidal J, et al. Studies on the sandfly fauna (Diptera: Psychodidae: Phlebotominae) from transmission areas of American cutaneous leishmaniasis in state of Acre, Brazil. Mem Inst Oswaldo Cruz. 2008; 103(8): 760-7.

da Graça GC, Volpini AC, Romero GAS, de Oliveira Neto MP, Hueb M, Porrozzi R, et al. Development and validation of PCR-based assays for diagnosis of American cutaneous leishmaniasis and identification of the parasite species. Mem Inst Oswaldo Cruz. 2012; 107(5): 664-74.

de Araujo-Pereira T, de Pita-Pereira D, Boité MC, Melo M, da Costa-Rego TA, Fuzari AA, et al. First description of Leishmania
(Viannia) infection in Evandromyia saulensis, Pressatia sp. and Trichophoromyia auraensis (Psychodidae: Phlebotominae) in a transmission area of cutaneous leishmaniasis in Acre state, Amazon Basin, Brazil. Mem Inst Oswaldo Cruz. 2017; 112(1): 75-8.

de Araujo-Pereira T, Fuzari AA, Andrade Filho JD, de Pita-Pereira D, Britto C, Brazil RP. Sand fly fauna (Diptera: Psychodidae: Phlebotominae) in an area of leishmaniasis transmission in the municipality of Rio Branco, state of Acre, Brazil. Parasit Vectors. 2014; 7: 360.

de Pita-Pereira D, Alves CR, Souza MB, Brazil RP, Bertho A, Barbosa A, et al. Identification of naturally infected Lutzomyia intermedia and Lutzomyia migonei with Leishmania (Viannia) braziliensis in Rio de Janeiro (Brazil) revealed by a PCR multiplex non-isotopic hybridization assay. Trans R Soc Trop Med Hyg. 2005; 99(12): 905-13.

Martins AV, Silva JE. Notas sobre os flebotomíneos do estado do Acre, com a descrição de duas espécies novas (Diptera, Psychodidae). Rev Bras Biol. 1964; 24: 127-38.

Oliveira AFJ, Teles CBG, Medeiros FM, Camargo LMA, Pessoa FAC. Description of Trichophoromyia ruifreitasi, a new phlebotomine species (Diptera, Psychodidae) from Acre state, Brazilian Amazon. Zookeys. 2015; 526: 65-73.

Silva-Nunes M, Cavasini CE, Silva NS, Galati EAB. Epidemiologia da Leishmaniose Tegumentar e descrição das populações de flebotomíneos no município de Acrelândia, Acre, Brasil. Rev Bras Epidemiol. 2008; 11(2): 241-51.

Teles CBG, dos Santos APA, Freitas RA, de Oliveira AFJ, Ogawa GM, Rodrigues MS, et al. Phlebotomine sandfly (Diptera: Psychodidae) diversity and their Leishmania DNA in a hot spot of American Cutaneous Leishmaniasis human cases along the Brazilian border with Peru and Bolivia. Mem Inst Oswaldo Cruz. 2016; 111(7): 423-32.

Teles CBG, Freitas RA, Oliveira AFJ, Ogawa GM, Cavalcante EA, Medeiros JF, et al. Description of a new phlebotomine species (Diptera: Psychodidae, Phlebotominae) and new records of sand flies from the state of Acre, Northern Brazil. Zootaxa. 2013; 3609(1): 85-90.

Valdivia HO, de los Santos MB, Fernández R, Baldeviano GC, Zorrilla VO, Vera H, et al. Natural Leishmania infection of Lutzomyia (Trichophoromyia) auraensis in Madre de Dios, Peru, detected by a fluorescence resonance energy transfer-based real-time polymerase chain reaction. Am J Trop Med Hyg. 2012; 87(3): 511-7.

Young DG, Ducan NA. Guide to the identification and geographic distribution of Lutzomyia sandflies in Mexico, the West Indies, Central and South America (Diptera: Psychodidae). Mem Am Entomol Inst. 1994; 54: 1-881.

Thais de Araujo-Pereira Daniela de Pita-Pereira Laboratório de Biologia Molecular e Doenças Endêmicas Instituto Oswaldo Cruz - Fiocruz Rio de Janeiro, RJ, Brasil

Mariana Côrtes Boité Laboratório de Pesquisa em Leishmaniose Instituto Oswaldo Cruz - Fiocruz Rio de Janeiro, RJ, Brasil Myllena Melo Taiana Amancio da Costa-Rego Laboratório de Biologia Molecular e Doenças Endêmicas Instituto Oswaldo Cruz - Fiocruz Rio de Janeiro, RJ, Brasil

Andressa Alencastre Fuzari Reginaldo Peçanha Brazil

Laboratório de Doenças Parasitárias Instituto Oswaldo Cruz - Fiocruz Rio de Janeiro, RJ, Brasil

Constança Britto

Laboratório de Biologia Molecular e Doenças Endêmicas Instituto Oswaldo Cruz - Fiocruz Rio de Janeiro, RJ, Brasil 